\title{
SATELLITES IN MILKY-WAY-LIKE HOSTS: ENVIRONMENT DEPENDENCE AND CLOSE PAIRS
}

\author{
Roberto E. GonZÁlez ${ }^{1,2}$, Andrey V. Kravtsov ${ }^{1,2,3}$, and Nickolay Y. Gnedin ${ }^{1,2,4}$ \\ ${ }^{1}$ Department of Astronomy \& Astrophysics, The University of Chicago, Chicago, IL 60637, USA; regonzar@oddjob.uchicago.edu \\ ${ }^{2}$ Kavli Institute for Cosmological Physics, The University of Chicago, Chicago, IL 60637, USA \\ ${ }^{3}$ Enrico Fermi Institute, The University of Chicago, Chicago, IL 60637, USA \\ ${ }^{4}$ Particle Astrophysics Center, Fermi National Accelerator Laboratory, Batavia, IL 60510, USA \\ Received 2013 January 13; accepted 2013 April 14; published 2013 May 29
}

\begin{abstract}
Previous studies showed that an estimate of the likelihood distribution of the Milky Way (MW) halo mass can be derived using the properties of the satellites similar to the Large and Small Magellanic Clouds (LMC and SMC). However, it would be straightforward to interpret such an estimate only if the properties of the Magellanic Clouds (MCs) are fairly typical and are not biased by the environment. In this study, we explore whether the environment of the MW affects the properties of the SMC and LMC such as their velocities. To test for the effect of the environment, we compare velocity distributions for MC-sized subhalos around MW hosts in a sample selected simply by mass and in the second sample of such halos selected with additional restrictions on the distance to the nearest cluster and the local galaxy density, designed to mimic the environment of the Local Group (LG). We find that satellites in halos in the LG-like environments do have somewhat larger velocities, as compared to the halos of similar mass in the sample without environmental constraints. For example, the fraction of subhalos matching the velocity of the LMC is $23 \% \pm 2 \%$ larger in the LG-like environments. We derive the host halo likelihood distribution for the samples in the LG-like environment and in the control sample and find that the environment does not significantly affect the derived likelihood. We use the updated properties of the SMC and LMC to derive the constraint on the MW halo mass of $\log \left(M_{200} / M_{\odot}\right)=12.06_{-0.19}^{+0.31}(90 \%$ confidence interval). We also explore the incidence of close pairs with relative velocities and separations similar to those of the LMC and SMC and find that such pairs are quite rare among $\Lambda \mathrm{CDM}$ halos. Only $2 \%$ of halos in the MW mass range have a relatively close pair $(\Delta r<40 \mathrm{kpc}$ and $\Delta s<160 \mathrm{~km} \mathrm{~s}^{-1}$ ) of subhalos with circular velocities $v_{\text {circ }}>50 \mathrm{~km} \mathrm{~s}^{-1}$. Pairs with masses and separations similar to those of the LMC and SMC $\left(\Delta r_{\mathrm{MC}}=23.4 \pm 10 \mathrm{kpc}\right.$ and $\left.\Delta s_{\mathrm{MC}}=128 \pm 32 \mathrm{~km} \mathrm{~s}^{-1}\right)$ are found only in one out of $\approx 30,000 \mathrm{MW}$-sized halos. Interestingly, the halo mass likelihood distribution for host halos constrained to have MC-like close pairs of subhalos is quite different from the global likelihood from which the MW halo mass constraint discussed above was derived. Taking into account the close separation of the MCs in the Busha et al. method results in the shift of the MW halo mass estimate to smaller masses, with the peak shifting approximately by a factor of two.
\end{abstract}

Key words: dark matter - Galaxy: fundamental parameters - Galaxy: halo - Magellanic Clouds

Online-only material: color figures

\section{INTRODUCTION}

In the cold dark matter (CDM) scenario the Milky Way (MW) halo is formed by accretion and disruption of smaller halos, and some of them survived this process as self-bound substructures or subhalos orbiting around the host halo (e.g., Klypin et al. 1999b; Moore et al. 1999; see Kravtsov 2010 for a recent review). The majority of these subhalos are likely devoid of stars, but some of them are massive enough to host dwarf galaxies (e.g., Kauffmann et al. 1993; Klypin et al. 1999b; Bullock et al. 2000; Kravtsov et al. 2004; Font et al. 2011). The MW and its satellites offer a unique laboratory for testing CDM predictions for halo formation and associated substructure because properties of many of the satellites have been studied in detail in observations, including proper motions and rotation curves (e.g., Kallivayalil et al. 2006a, 2006b).

It is still debated how typical the satellite population of the MW is, but both theoretical models and observations indicate that the incidence of the Magellanic Clouds (MCs) satellites is rare for MW-sized galaxies. Semi-analytic galaxy formation models predict that only $\simeq 10 \%$ of the MW-sized galaxies have satellites as bright as the Large Magellanic Cloud (LMC; e.g., Kauffmann et al. 1993; Koposov et al. 2009). Boylan-Kolchin et al. (2011) used a large sample of MW-sized halos extracted from the Millennium-II simulation and found that $20 \%$ of MW-mass halos host an LMC or Small Magellanic Cloud (SMC), and only $\sim 2.5 \%$ host both MCs. A similar frequency was found by Busha et al. (2011b) using the Bolshoi simulation of the concordance cosmology and abundance matching ansatz to assign stellar masses to halos and subhalos. In observations, James \& Ivory (2011) searched for star-forming satellites around 143 luminous spiral galaxies and found that two-thirds of central galaxies have no satellites down to luminosity and star formation rates well below those of the MCs. Using the Sloan Digital Sky Survey (SDSS), Liu et al. (2011) computed the occurrence of satellites similar to the MCs in luminosity around MW-sized galaxies and found that $11 \%$ have one and only $3.5 \%$ have two MCs within a radius of $150 \mathrm{kpc}$. Similar values for the frequency of MCs are found in the SDSS in a recent study by Tollerud et al. (2011), and in the GAMA survey by Robotham et al. (2012). What makes MCs even more peculiar is the fact that they are likely to be an interacting pair (see Section 4.3) and have rather high velocities with respect to the MW.

One caveat in this debate is that some satellite properties depend sensitively on the MW halo mass (e.g., Wang et al. 2012). Although there are several methods to constrain the MW halo mass (see, e.g., Busha et al. 2011a; Deason et al. 2012 and references therein), it is still quite uncertain. In this study, 
we focus on the methods that use satellite properties to constrain the MW halo mass. In particular, as shown by Busha et al. (2011a, hereafter B11), the MW host halo mass likelihood can be computed using the observed MCs' properties and a statistically representative sample of CDM halos to evaluate the likelihood that a given halo would have each or all MCs' properties, such as circular velocity, host halo distance, and velocity within the host. Such approach provides a new interesting constraint on the MW virial mass, but there are certain questions that need to be addressed to assess its reliability and interpretation. One of such questions is whether the environment of the Local Group (LG) biases properties of the MW satellite population, and MCs in particular. We explore this and other related questions in more detail in this paper.

Another important question is the origin and dynamics of the MCs. The accretion history of the MCs is still not clear, the presence of the Magellanic Stream (MS hereafter), a filament of gas extending $150^{\circ}$ across the sky, with an apparent spatial and chemical association with the MCs, is interpreted as a tidal tail (see Besla et al. 2012 and references therein). There are several clues which indicate that the MCs are bound, and have been interacting recently (Diaz \& Bekki 2012; Besla et al. 2012), and there is also some evidence of the SMC stars accreted onto the LMC (Olsen et al. 2011; Casetti-Dinescu et al. 2012). Close satellite pairs are rare in nearby MW-sized galaxies (Robotham et al. 2012; James \& Ivory 2011). Proper motion measurements for the MCs (Kallivayalil et al. 2006a, 2006b) indicate that they have high velocities not aligned with the MS, and suggest that the MCs could be bound and on their first or second pericenter passage into the MW (Besla et al. 2007, 2010, 2012). Other studies also suggest that the MCs were accreted in the same system (D'Onghia \& Lake 2008; Nichols et al. 2011). B11 estimated from the Bolshoi simulation that, for a typical MW-sized host, there is a $\simeq 72 \%$ probability that the MCs were accreted within the last Gyr. Boylan-Kolchin et al. (2011) and Besla et al. (2012) also favor the late accretion scenario for the MCs.

This paper is organized as follows. We describe the simulation and halo catalogs used in our study in Section 2, while in Section 3 we describe the data samples for our MW, MCs, and LG analogs. We present the MW halo mass likelihood in different environments and under different assumptions on whether MCs are independent velocity samples in Sections 4 and 4.3. We present discussion and our conclusions in Section 5.

\section{SIMULATION AND HALO CATALOGS}

To carry out our analysis we use halos extracted from the Bolshoi simulation (Klypin et al. 2011), which followed the evolution of $2048^{3}$ particles in $250 \mathrm{~h}^{-1} \mathrm{Mpc}$ cubic volume assuming concordance flat $\Lambda \mathrm{CDM}$ cosmology with parameters $\Omega_{\mathrm{m}}=1-\Omega_{\Lambda}=0.27, \Omega_{\mathrm{b}}=0.0469, h=H_{0} /(100)=$ $0.7, \sigma_{8}=0.82$, and $n_{\mathrm{s}}=0.95$, compatible with combined constraints from the Wilkinson Microwave Anisotropy Probe, Baryonic Acoustic Oscillations (BAO), supernovae, and cluster abundance (Komatsu et al. 2011). The high spatial and mass resolution and relatively large volume make the Bolshoi simulation well suited for providing a base halo sample for our study.

The halo catalog we used was constructed using the Bound Density Maxima halo finder (Klypin \& Holtzman 1997; Klypin et al. 1999a), which identified 2,285,977 halos down to the resolution completeness limit of $v_{\text {circ }} \approx 50 \mathrm{~km} \mathrm{~s}^{-1}$ (corresponding to $\approx 110$ particles or $M_{200} \approx 1.7 \times 10^{10} M_{\odot}$ ). The circular velocity $v_{\text {circ }}$ is the maximum of the circular velocity profile $v_{\text {circ }}(r)=\sqrt{G M(<r) / r}$. The parameters of the halo finder were set such that the density maxima are not allowed to be closer than $10 h^{-1} \mathrm{kpc}$, and the finder keeps only the most massive density maximum if that happens. Halo center is identified with the particle location which has the largest local density, and bulk halo velocity is computed as the average velocity of the 30 closest neighbors of the central particle. The algorithm computes a number of halo properties after an iterative procedure to remove unbound particles.

Throughout this paper we define halo mass, $M_{200}$, as the mass enclosed in a sphere of radius $R_{200}$ with a density 200 times the critical density of the universe at the redshift of analysis. Another common mass definition is $M_{\text {vir }}$, the mass within the radius enclosing the mean overdensity of 358 with respect to the mean density of the universe (or overdensity of $358 \times 0.27 \approx 97$ with respect to the critical density; Bryan \& Norman 1998). In the Bolshoi simulation, we find a relation of $M_{\mathrm{vir}} / M_{200}=1.21$ for host halos of $v_{\text {circ }} \approx 220 \mathrm{~km} \mathrm{~s}^{-1}$.

We use MW-sized host halos in the Bolshoi catalogs to search for subhalos with velocities and positions similar to those of the MCs. However, the positions of satellites change on short timescales due to their motion along their orbits. Therefore, to increase statistics, we stack the halo catalogs of several simulation snapshots close to $z=0$ separated by $\Delta a=0.003$ $(\sim 42$ Myr at $z=0)$. During the time interval between snapshots, a typical MC should move $\sim 15 \mathrm{kpc}$ along its trajectory. We stack satellites of the last 30 snapshots, and the total time difference between the first and last snapshot is $\sim 1.3 \mathrm{Gyr}$ ( or $\Delta z<0.1$ ), so we can neglect any evolution effect in MW mass halos (MuñozCuartas et al. 2011; Diemer et al. 2013; Cuesta et al. 2008).

For a given MW-sized halo at $z=0$ we should, in principle, consider the last $N$ simulation outputs to trace trajectories of all satellites and check if they match MCs' constraints at some time in the recent past. However, this is computationally expensive. Instead, in our analysis we consider each snapshot as an independent realization of halo properties, which effectively increases the simulation sample by a factor of $N$. In this approximation, we neglect any correlation in the positions and velocities of satellites between snapshots. For example, in the extreme case of a purely circular orbit, a single LMC or SMC analog would be counted $N$ times. However, for realistic eccentric orbits such double counting is quite rare, especially for satellites with relatively small radial distances to host centers similar to those of the SMC and LMC. On the other hand, we have a large number of hosts and satellites in each snapshot, and the randomness of their orbital configuration produces consistent distributions and average fraction of satellites matching any given set of constraints, for any number of snapshots selected. We have tested for the double counting effects using different number of snapshots, $N$, computing the distribution of satellites matching several set of constraints, and we have found no significant differences in the properties of satellites for $N \lesssim 30$.

\section{HALO SAMPLES AND OBSERVATIONAL CONSTRAINTS}

In our analysis we use the following three main observational measurements.

\subsection{The Milky Way Halo Mass}

To select MW analogs from the Bolshoi-derived halo catalogs, we select halos within the broad mass range $M_{200 c}=$ 0.8-2.9 $\times 10^{12} M_{\odot}$, which covers the range of current 
observational constraints: e.g., using $\mathrm{HI}$ gas distribution (Kalberla et al. 2007), kinematics of stars (Sofue et al. 2009; Xue et al. 2008; Gnedin et al. 2010; Wilkinson \& Evans 1999; Deason et al. 2012; Kafle et al. 2012), satellite dynamics (Watkins et al. 2010), escape velocity (Smith et al. 2007), and timing argument (Li \& White 2008). There are $(\sim 57,000)$ host halos in this mass range in the Bolshoi simulation at $z=0$, which contain $\sim 115,000$ subhalos with $v_{\text {circ }}>50 \mathrm{~km} \mathrm{~s}^{-1}$.

\subsection{Magellanic Cloud Analogs}

We follow Busha et al. (2011a) and use the following observed properties of the MCs to select appropriate MC analogs among subhalos in the MW halo analogs and to constrain the halo mass of the MW: the distance to the host center $r_{0}$, the total speed relative to the host center $s$, and the subhalo circular velocity $v_{\text {circ }}$. For these quantities, we use recent Hubble Space Telescope measurements by Kallivayalil et al. (2013): $v_{\text {circ }}=$ $76.1 \pm 7.6 \mathrm{~km} \mathrm{~s}^{-1}, r_{0}=50 \pm 5 \mathrm{kpc}, s=321 \pm 24 \mathrm{~km} \mathrm{~s}^{-1}$ for the LMC, and $v_{\text {circ }}=60 \pm 5 \mathrm{~km} \mathrm{~s}^{-1}, r_{0}=60 \pm 5 \mathrm{kpc}$, $s=217 \pm 26 \mathrm{~km} \mathrm{~s}^{-1}$ for the SMC. The halos hosting the MCs' analogs are required to have at least two subhalos with $v_{\text {circ }}>50 \mathrm{~km} \mathrm{~s}^{-1}$ and we will consider only the two subhalos with the largest circular velocities. ${ }^{5}$

Note that $r_{0}$ errors are inflated from their actual observational values to improve the statistics of the sample of the MC analogs. The range of $r_{0}$ we use corresponds to a typical radial displacement of subhalos along its orbit between consecutive snapshots. These parameters are used everywhere in this paper, except in Section 4.2, where we use a different definition for the MCs' analogs assuming a single bound system, and in Section 5, where we include two additional constraints: the relative separation and velocity of the clouds.

\subsection{The Local Group Analogs}

The MW is not an isolated galaxy, but is located in a pair with M31 ( $\approx 770 \mathrm{kpc}$ away; McConnachie et al. 2005; Ribas et al. 2005) and is surrounded by a number of smaller galaxies, collectively known as the LG of galaxies. On larger scales the environment of the LG is rather low density: in a sphere of $50 \mathrm{Mpc}$ radius around the $\mathrm{LG}$, the estimated density is $\sim 3$ times lower than average (Karachenstev 2012), while in a sphere of $5 \mathrm{Mpc}$ the density around the LG is close to the mean density of the universe (Klypin et al. 2003; Karachentsev 2005). In addition, the nearest cluster to the LG is the Virgo Cluster 16.5 Mpc away (Mei et al. 2007).

To explore whether the environment of the LG on different scales affects the statistics of the MC analogs, we derive several MW analog samples that mimic different aspects of the real MW environment: the host halos in the P sample have an M31-sized companion in relative isolation with no other large neighbor; host halos in the LGP sample are a subset of halo pairs from the $\mathrm{P}$ sample, but with additional environmental constraints designed to more closely mimic the LG environment; finally, host halos in the S sample include all host halos that are not included in the LGP and P samples. These sample definitions and naming conventions will be used for both host halos in the MW halo mass range (Section 3.3) and halo samples in a wide range of masses (Section 4). Note that for the P and LGP samples the mass of the M31-like companion is always fixed to the same

5 In B11, the MCs' analogs are selected in hosts that have exactly two subhalos with $v_{\text {circ }}>50 \mathrm{~km} \mathrm{~s}^{-1}$. We will explore the effects of this difference in the Results section. mass range, even as its MW analog halo mass is varied within a wider range.

Below the sample definitions are described in more detail.

The P sample: the host halos are required to have a companion similar to M31, which we define using the same mass range of $M_{200 c}=0.8-2.9 \times 10^{12} M_{\odot}$ (e.g., Watkins et al. 2010; Evans et al. 2000). The pairs are required to be relatively isolated and not part of a triplet or a larger group. As a quantitative isolation criterion for the pair we use the force constraint $F_{i \text {,com }}<\kappa F_{12}$, where $F_{i, \text { com }}$ is the gravitational force between the pair and any neighbor halo $i$ within a $5 h^{-1} \mathrm{Mpc}$ radius of the pair center of mass, $F_{12}$ is the force between halos in the pair, and $\kappa$ is a constant parameter. The isolation criterion becomes increasingly strict for decreasing values of $\kappa$. The MW and M31 do not have massive neighbors within $5 \mathrm{Mpc}$, and should thus have $\kappa<0.1$. The actual value of $\kappa$ is, however, uncertain and we explore a range of values in the further analysis. We use $\kappa=0.1,0.15,0.2$, and 0.25 which results in 205, 378, 598, and 810 LG-like halo pairs, respectively. From the a posteriori analysis of satellite properties, the results are largely consistent for different $\kappa$ values and we thus use $\kappa=0.2$ in this paper to maximize the statistics of the MW hosts. The 598 LG-like halo pairs we found using $\kappa=0.2$ represent $\sim 2 \%$ of all halos in the MW mass range, and $\sim 80 \%$ of these pairs are bound under the two-body point mass energy approximation.

The LGP sample of halos is a subset of halos in the $\mathrm{P}$ sample with additional constraints on the local and global environment to more closely mimic the environment of the LG. Namely, we require that halos in this sample do not have a cluster-sized halo more massive than $M_{200}=1.5 \times 10^{14} M_{\odot}$ within $12 \mathrm{Mpc}$, consistent with lower limit values for the Virgo Cluster mass and distance estimates (e.g., Fouqué et al. 2001; Nulsen \& Bohringer 1995 and references therein). For the local environment we compute the galaxy density field using the Voronoi tessellation (hereafter VT) on halo positions and masses (similar to the method of Schaap \& van de Weygaert 2000; González \& Padilla 2010). The VT partitions the volume into cells, where each cell is associated with a single host halo. The shape and volume of each cell is defined by the distribution of halo neighbors. The adaptive local density can be computed using the local cell volume around each halo and the enclosed halo mass, but instead we also use neighbors cell volumes and masses to compute the average; in this way, we generate a smooth density field where the typical number of direct neighbors around a halo is $\approx 14$. We use only the host halos with masses higher than $M_{200}=1.5 \times 10^{10} M_{\odot}$ for density computation, and define the local overdensity as $\delta=(\rho-\langle\rho\rangle) /\langle\rho\rangle$, where $\langle\rho\rangle$ is the mean of the density distribution. The local density of the LG is not well constrained, but the abundance of luminous galaxies within 3-6 Mpc is close to the average density of galaxies in the local universe (Karachentsev 2005). Another local environment constraint we include is the distance to the M31-like pair companion.

Figure 1 shows the distribution of the local density, the distance to the closest Virgo-sized halo, and the distance to the M31-sized pair companion for the MW analog halos in the $\mathrm{P}$ sample, and the cuts we impose to define the LGP sample. Specifically, we select only halos with smaller local overdensity (i.e., $\delta=1.04$ ) to avoid systems located in the highest density decile. The mean overdensity for the $\mathrm{S}$ sample is located at $\log (\delta+1) \sim 0$, and for the LGP sample it is located $0.5 \mathrm{dex}$ lower.

We exclude halos with Virgo-sized neighbors closer than $12 \mathrm{Mpc}$ and require that the distance to the pair companion 

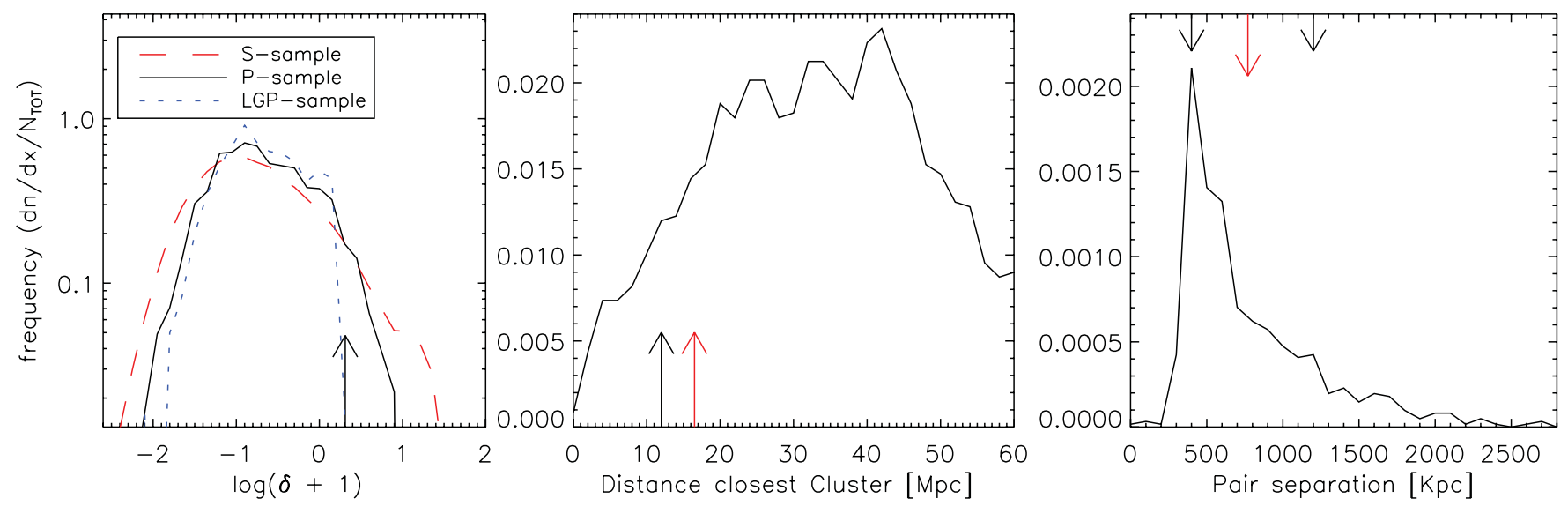

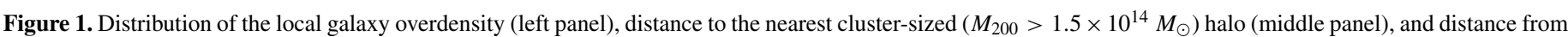

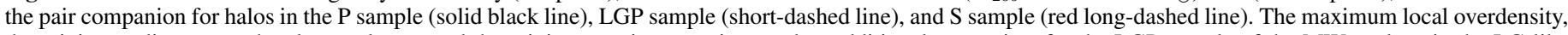

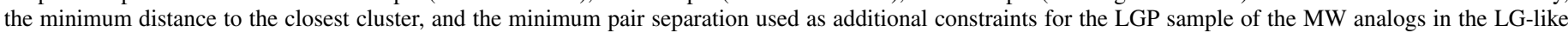

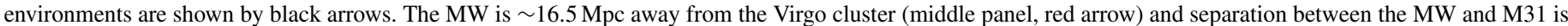
$\sim 770 \mathrm{kpc}$ (right panel, red arrow).

(A color version of this figure is available in the online journal.)

is in the range $0.4<\Delta r<1.2 \mathrm{Mpc}$ to avoid close, possibly merging, pairs, but to include pairs with separations similar to the actual distance between MW and M31. These constraints eliminate about one-third of the halos from the P sample; the halos in the LGP sample are $\approx 1.3 \%$ of the total number of host halos in the MW halo mass range. This indicates that the LG environment of the MW is rather rare for halos of this mass, the fact also indicated by the "coldness" of the local velocity field of galaxies (e.g., Klypin et al. 2003).

The figure shows that the $\mathrm{P}$ sample halos are located in environments with an overdensity distribution similar to the S-sample halos, but have a narrower distribution due to the force constraint and the chosen $\kappa=0.2$ value, which eliminates responsible pairs close to other larger halos and single isolated halos.

\section{THE MW HALO MASS ESTIMATE}

In the context of the B11 method, in order to estimate the halo mass of the MW from the properties of the MCs, such as circular velocity, velocity, and position relative to the center, we explore two aspects which can affect the mass estimate: (1) the environment, in particular whether differences in the environment correspond to the differences in the subhalo populations of halos, and (2) whether it matters if MCs are treated as two independent dynamical samples or a single tracer (a bound pair sharing a common translational motion of their center of mass).

\subsection{Effect of the Environment}

To test for possible effects of the MW environment on subhalo statistics, we extract all subhalos with $v_{\text {circ }}>50 \mathrm{~km} \mathrm{~s}^{-1}$, and compute their distance to the host center $r_{0}$ and the total speed relative to host $s$. Subhalos very close to the host center $\left(r_{0}<20 h^{-1} \mathrm{kpc}\right)$ are removed to avoid artifacts or resolutionrelated problems in the halo identification procedure.

Figure 2 shows the abundance of subhalos with $v_{\text {circ }}>$ 50,60 , and $70 \mathrm{~km} \mathrm{~s}^{-1}$ in four mass ranges around $10^{12} M_{\odot}$. As expected, the average number of subhalos increases with increasing host halo mass and decreases for increasing subhalo circular velocities. The figure shows that there is no significant difference in the abundances of subhalos in hosts of the $\mathrm{S}$ and

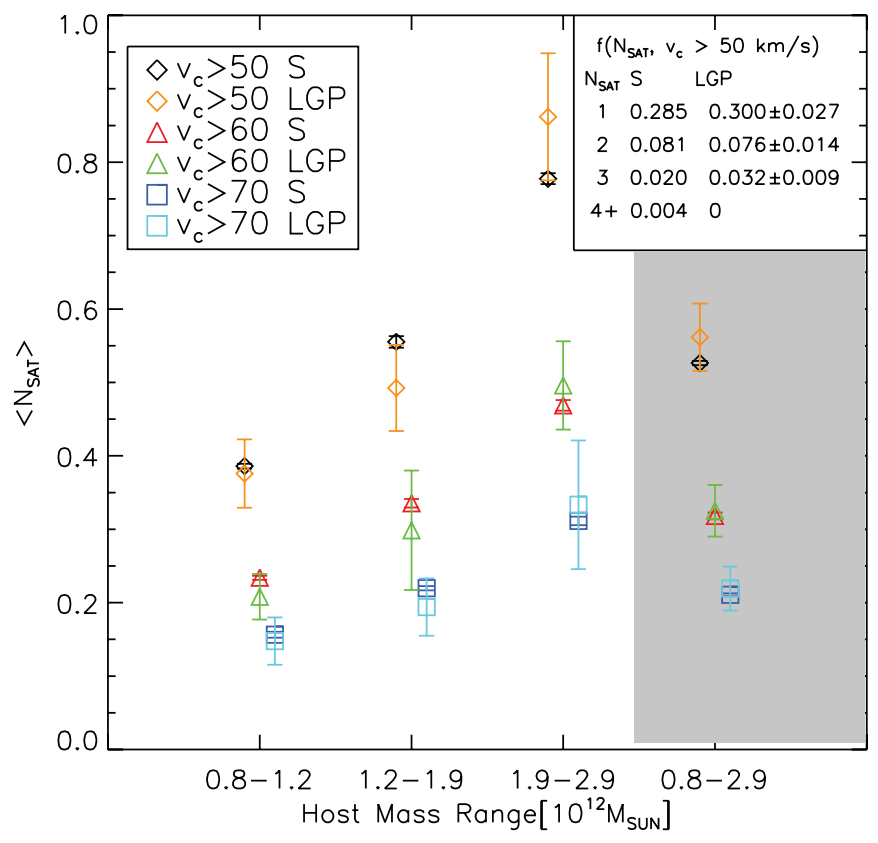

Figure 2. Average number of satellites in the $S$ and LGP samples for halos in different mass ranges around the most likely range of the MW halo mass, and satellite circular velocity ranges defined in legend. The error bars of the mean values are computed using the jackknife method. Top right legend quotes the fractions of halos with $N_{\text {SAT }}$ members for subhalos with $v_{\text {circ }}>50 \mathrm{~km} \mathrm{~s}^{-1}$, and host halo masses in the range $0.8 M_{\odot}<M_{200}<2.9 \times 10^{12} M_{\odot}$.

(A color version of this figure is available in the online journal.)

LGP samples, which means that the LG environment does not appreciably affect the abundance of the massive subhalos in the MW-sized hosts.

Figure 3 shows the distributions of the total subhalo velocity in the frame of their hosts for halos in the S and LGP samples. The fraction of satellites with velocities comparable to that of the LMC is $23 \% \pm 2 \%$ higher in the LGP sample than in the $\mathrm{S}$ sample (there is no significant change in the distribution at the SMC speed). Therefore, the fraction of satellites matching the velocity of the LMC is somewhat enhanced in the LG-like environments. This can affect the MW mass estimate because LMC velocity is the main constraint driving the mass likelihood distribution to larger masses (see B11 and Figure 5). 


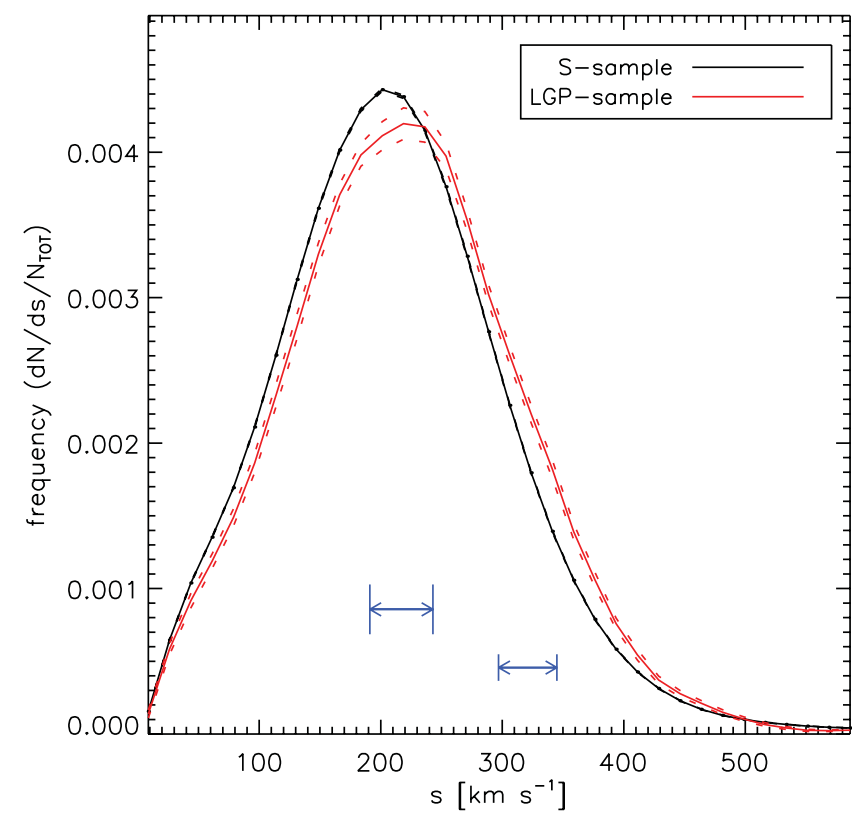

Figure 3. Distributions of the total subhalo velocity in the frame of their host halo for hosts in the S and LGP samples. The blue arrows indicate the velocities of the SMC and LMC. The uncertainty of the distribution for the LGP sample is shown by the dashed lines (estimated using the jackknife method). The environment has a small but statistically significant effect on the distribution of velocities: the fraction of subhalos with the LMC velocity in the LGP sample is $23 \% \pm 2 \%$ higher than in the S sample.

(A color version of this figure is available in the online journal.)

We have tested whether the choice of $\kappa$ parameter used in the isolation criterion (see Section 3.3) influences the velocity distribution and have found that the result velocity distribution is almost the same for values of $\kappa$ in the range $0.1<\kappa<0.25$, with a weak trend toward higher fraction of subhalos in the LGP sample matching the velocities of the LMC and SMC for lower $\kappa$ values. The magnitude of the trend, however, is comparable to the uncertainties in the velocity distribution.

In Figure 4, we show the fraction of subhalos with total velocities relative to the host center similar to those of the LMC and SMC for host halos in different mass ranges around $M_{200}=$ $10^{12} M_{\odot}$. There is a small enhancement in the frequency at the LMC velocity, but no difference at the SMC velocity. In the LGP sample the fraction of host halos with subhalos matching the LMC velocity is larger by $29 \% \pm 10 \%, 16 \% \pm 4 \%$, and $10 \% \pm 2 \%$ than in the $\mathrm{S}$ sample for $0.8-1.2,1.2-1.9$, and 1.9-2.9 $\times 10^{12} M_{\odot}$ mass ranges, respectively.

We present the likelihood distribution for the MW halo mass from the constraints from the MC properties in Figure 5. To compute the likelihood we used the $\mathrm{S}$ and LGP samples constructed without any restriction on the $M_{200}$ mass of halos. In addition to the likelihood for all of the constraints combined for $\mathrm{S}$ sample only, we also show likelihoods resulting from using only one of the constraints for both S and LGP samples. In particular, we present the likelihood for the constraint that host halos have exactly two subhalos with $v_{\text {circ }}>50 \mathrm{~km} \mathrm{~s}^{-1}$ with all other constraints (circular velocity, radial distance, and total velocity) applied after this condition to compare to the results of B11.

We chose not to enforce the two-satellite condition in our estimate of the likelihood, instead we allow the host halo to have any number of satellites to increase the sample size, but we will consider only the two largest ones. The mass estimates obtained with and without enforcing the two-satellite condition

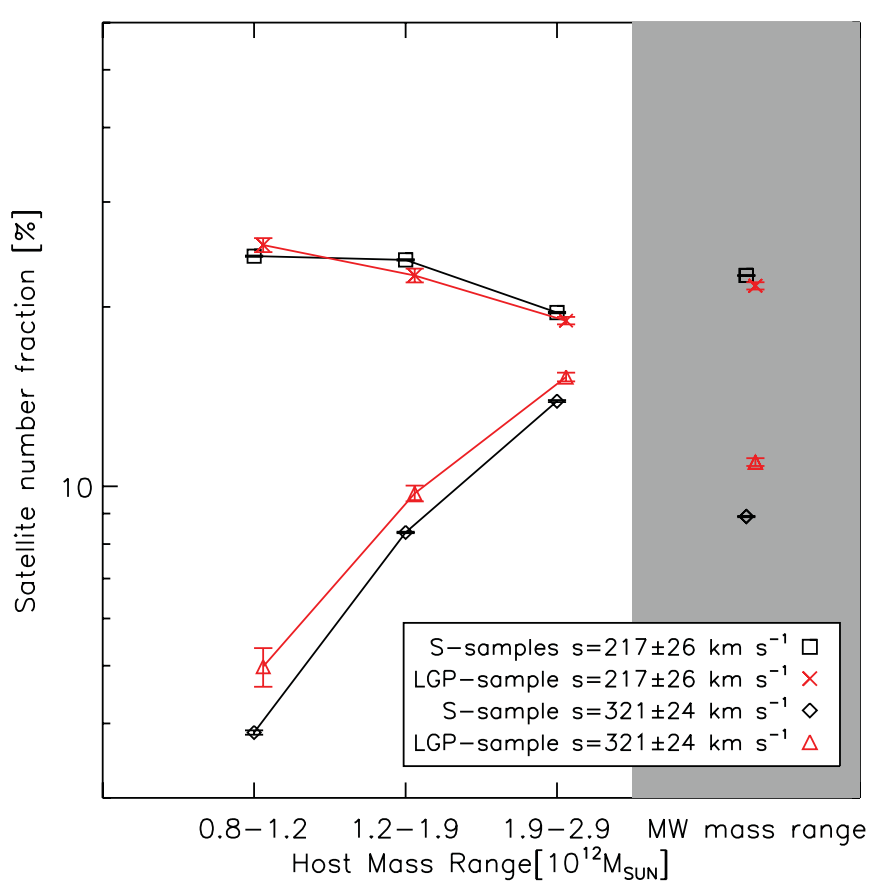

Figure 4. Fraction of subhalos matching the velocity of the SMC and LMC in the $\mathrm{S}$ (black symbols and lines) and LGP (red symbols and lines) samples for three different halo mass ranges around $M_{200}=10^{12} M_{\odot}$ (the first three connected bins) and the total mass range used to define the MW halo analogs (the gray shaded region).

(A color version of this figure is available in the online journal.)

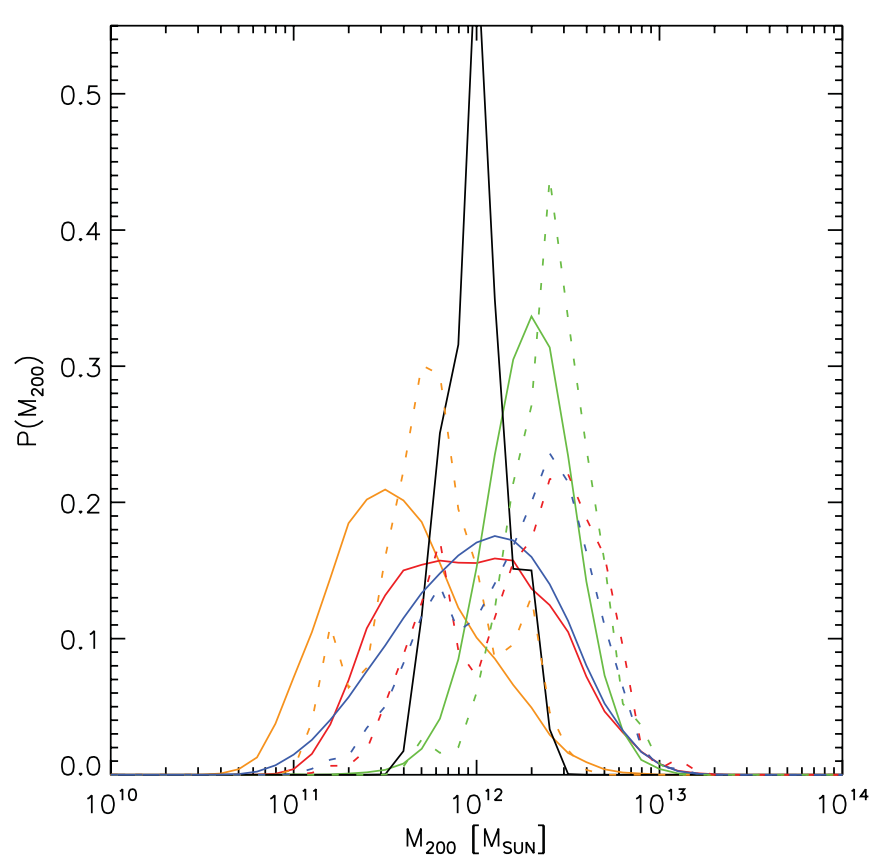

Figure 5. Likelihood distribution for the MW halo mass (black line), $M_{200}$, in the S sample based on the properties of the LMC and SMC allowing the full halo mass range instead of the range $M_{200}=0.8-2.9 \times 10^{12} M_{\odot}$. Lines of different colors show likelihoods when only one constraint is used: exactly two subhalos with $v_{c}>50 \mathrm{~km} \mathrm{~s}^{-1}$ (blue line), circular velocities of the MCs (red line), radial positions of the LMC and SMC (orange line), and total velocity relative to the host center of the LMC and SMC (green line). The dotted lines are corresponding distributions for the LGP sample using the same color key, but we omit the distribution using all constraints together in this case due to the lack of matching systems. The MW mass estimate in the S sample is $\log \left(M_{200} / M_{\odot}\right)=12.06_{-0.19}^{+0.31}(90 \%$ confidence interval $)$.

(A color version of this figure is available in the online journal.) 
Table 1

Constraints on the Halo Mass of the Milky Way Using Different Host Samples and Properties of the LMC and SMC

\begin{tabular}{lcccrr}
\hline \hline Sample & $\begin{array}{c}\text { Any Number Subs } \\
\log \left(M_{200} / M_{\odot}\right)\end{array}$ & $\begin{array}{c}v_{\text {circ }}>50 \mathrm{~km} \mathrm{~s}^{-1} \\
68 \% \mathrm{c} . \mathrm{i} .\end{array}$ & $90 \% \mathrm{c.i}$. & $\begin{array}{c}\text { Exactly Two Subs } \\
\log \left(M_{200} / M_{\odot}\right)\end{array}$ & $\begin{array}{c}v_{\text {circ }}>50 \mathrm{~km} \mathrm{~s} \\
68 \% \mathrm{c} . \mathrm{i} .\end{array}$ \\
\hline S-sample & 12.06 & $+0.08-0.05$ & $+0.31-0.19$ & 12.03 & $+0.06-0.02$ \\
S-multi & 12.24 & $+0.13-0.20$ & $+0.25-0.25$ & 12.15 & $+0.34-0.17$ \\
LGP-multi & 12.44 & $+0.07-0.13$ & $+0.24-0.22$ & 12.26 & $+0.15-0.05$ \\
LMC only & 12.18 & $+0.10-0.10$ & $+0.33-0.23$ & 12.17 & $+0.14-0.06$ \\
S-bound & 12.13 & $+0.13-0.11$ & $+0.36-0.25$ & 12.09 & +0.30 \\
LGP-bound & 12.17 & $+0.16-0.19$ & $+0.30-0.34$ & 11.99 & $+0.11-0.11$ \\
& & & & $+0.31-0.24$ \\
\hline
\end{tabular}

Notes. Columns 2-4 show the constraints for the case when host halos are allowed to have any number of satellites with $v_{\text {circ }}>50 \mathrm{~km} \mathrm{~s}{ }^{-1}$, while Columns 5-7 show the corresponding constraints for the case when host halos are restricted to have exactly two satellites with $v_{\text {circ }}>50 \mathrm{~km} \mathrm{~s}^{-1}$. We present the mass errors corresponding to both the $68 \%$ and $90 \%$ confidence intervals from the derived likelihood distributions.

are presented in Table 1. The final mass estimate using all of the constraints together is only computed for the S sample, in which there are $40 \mathrm{MC}$-like satellite pairs. There are only three such satellite pairs in the LGP sample, and therefore we do not attempt to derive the total likelihood for this sample. However, given that the likelihood distributions for individual property constraints are similar for the S and LGP samples, we expect that the total likelihood is similar for the combined constraints as well.

The MW halo mass estimate for the $\mathrm{S}$ sample is $\log \left(M_{200} / M_{\odot}\right)=12.06_{-0.19}^{+0.31}(90 \%$ confidence interval $)$, which is in general agreement with the B11 result in the central value. We present results for different samples and constraints in Table 1, where we also include both the $68 \%$ and $90 \%$ confidence interval errors. Due to significant deviations of the likelihood from the log-normal form in the tails, the $90 \%$ errors are significantly larger than the $68 \%$ ones and we therefore chose to quote the $90 \%$ errors.

Note that there is a key methodological difference in the way the likelihood distribution was evaluated in B11 and in our analysis. In our mass estimate, we use all the constraints together simply as the distribution of halo properties that satisfy the constraints. This way any correlations between properties, such as the expected correlation between radial distance to the host center and total velocity, are taken into account automatically. B11, on the other hand, assume that the probability distributions for each constraint are independent (see their Equation (3)). If we multiply the probability distributions for radial, speed, and circular velocity constraints, we get an estimate of the MW mass which should be similar to the method of B11. For the S sample this gives $\log \left(M_{200} / M_{\odot}\right)=12.24_{-0.25}^{+0.25}$ and for the LGP sample $\log \left(M_{200} / M_{\odot}\right)=12.44_{-0.22}^{+0.24}$. Thus, treating constraints as independent results in a small overestimate of the mass.

\subsection{Dependence on the SMC and LMC Constraints}

The second key assumption of the B11 analysis is that the properties of the two MCs are not correlated. However, the MCs are likely a bound pair, and their velocities and radial positions can thus be expected to be correlated. We have tested whether this assumption affects the MW mass estimate by comparing results in the following two cases: (1) properties of the MCs are treated as independent and we compare the mass likelihood using both MCs, only the LMC, or only the SMC; (2) the MCs are considered to be a bound pair and we compute the likelihood using average properties of the pair, rather than properties of the two MCs independently. We explore the latter scenario in the next subsection.
For case 1 we compute the mass likelihood for the LMC and SMC independently and find $\log \left(M_{200} / M_{\odot}\right)=12.18_{-0.23}^{+0.33}$ using the LMC only, consistent with the result using both MCs discussed above. For the case when we use velocity of the SMC only, the mass is not well constrained as the likelihood extends to considerably lower masses. Thus, the MW mass estimate is dominated by the properties of the LMC, as is expected since it is more massive and has a higher velocity.

The fact that the main mass constraint comes from the satellite with the largest velocity is generic. If one considers the distribution of absolute magnitude of satellite velocities as a function of the radial distance to the host center, at any given $r$ the distribution is broad but has a sharp cutoff at the velocity close to the escape velocity of the host. It is this sharp cutoff that constrains the mass, and the constraint is due to the satellite with the largest absolute velocity at a given radius. This fact was recently used to constrain the halo mass of the MW with Leo I satellite by Boylan-Kolchin et al. (2013).

In principle, we can include additional properties of the LMC and SMC, such as their separation or relative velocity $(\approx 23 \mathrm{kpc}$ and $128 \pm 32 \mathrm{~km} \mathrm{~s}^{-1}$, respectively; Kallivayalil et al. 2006a, 2013), in the derivation of the mass constraint. However, this is difficult in practice because very few host halos in the Bolshoi simulation match all of the properties of the LMC and SMC. We examine the incidence of the MC-like close pairs of satellites in the next section.

Here we adopt a different approach, in which we assume that the LMC and SMC are a bound pair and can be considered as a single subhalo. We then consider the pair as a single velocity tracer and use the velocity of the center of mass of the pair as a constraint. We calculate the center-of-mass velocity assuming that the MCs move in the same direction and neglecting peculiar velocity around the center of mass: $\left\|\boldsymbol{s}_{\mathrm{cm}}\right\|=\left\|\boldsymbol{s}_{1} M_{1}+\boldsymbol{s}_{2} M_{2}\right\| /\left(M_{1}+M_{2}\right)=298 \pm 52 \mathrm{~km} \mathrm{~s}^{-1}$. We also treat the pair as a single subhalo with the circular velocity of $v_{\text {circ }}=85.4_{-10}^{+16} \mathrm{~km} \mathrm{~s}^{-1}$. The masses are evaluated numerically from the $M_{200}-v_{\text {circ }}$ relation for subhalos in the entire Bolshoi simulation. The asymmetry in the error range is because we include larger values for the LMC $v_{\text {circ }}$ (Olsen et al. 2011). Finally, we adopt the radial distance of the center of mass of $r_{\mathrm{cm}}=\left(r_{1} M_{1}+r_{2} M_{2}\right) /\left(M_{1}+M_{2}\right)=54 \pm 5 \mathrm{kpc}$.

Figure 6 shows the likelihood distribution for the MW halo mass estimated with such constraint for the S and LGP samples. We also include the mass estimate from Figure 5 for comparison. The halo mass in this case is constrained to be $\log \left(M_{200} / M_{\odot}\right)=12.13_{-0.25}^{+0.36}(90 \%$ confidence interval) in the S sample and $\log \left(M_{200}\right)=12.17_{-0.34}^{+0.30}$ in the LGP sample. We do not find any significant variation with environment in this 


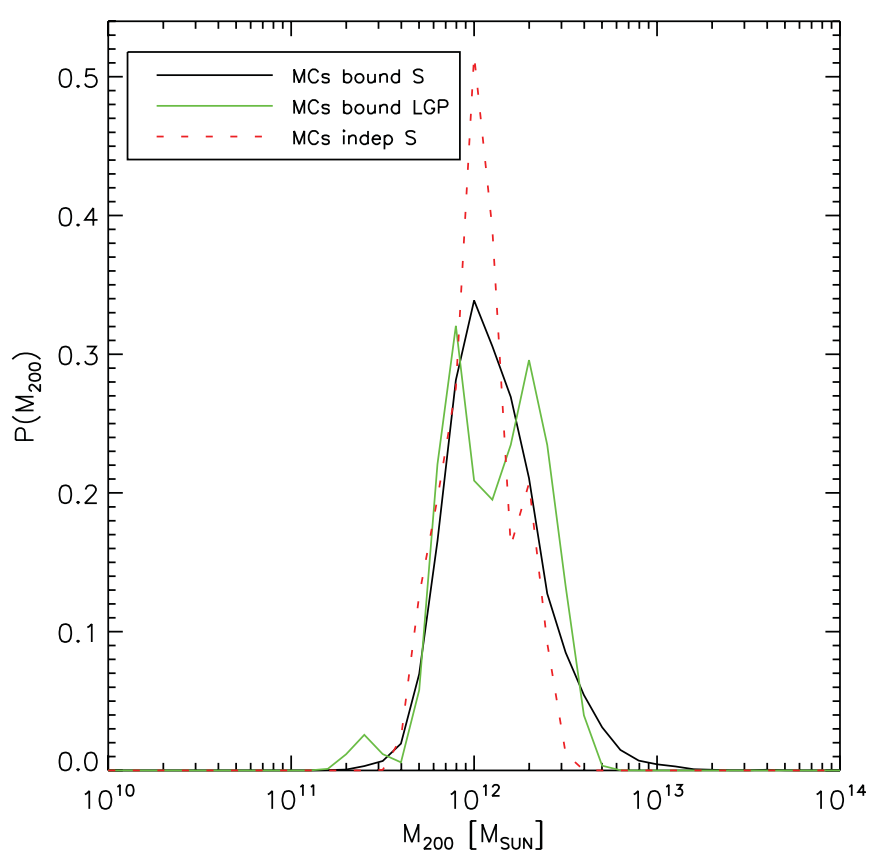

Figure 6. Likelihood distribution of the MW halo resulting from the SMC and LMC being considered a bound system corresponding to a single subhalo (with parameters quoted in the text). The distribution for the $\mathrm{S}$ sample is shown by the black solid line, and for the LGP sample by the green solid line. The corresponding halo mass constraints are $\log \left(M_{200} / M_{\odot}\right)=12.13_{-0.25}^{+0.36}$ and $\log \left(M_{200} / M_{\odot}\right)=12.17_{-0.34}^{+0.30}$ (errors correspond to the $90 \%$ confidence level) for the S and LGP samples, respectively. The mass estimate for the $\mathrm{S}$ sample from Figure 1 derived when properties of both MCs are used separately is shown for comparison by the dotted red line.

(A color version of this figure is available in the online journal.)

case, and the mass estimate errors are consistent with the case when we constrain the mass using both MCs as independent tracers. The only difference is that the use of the MCs as a single tracer eliminates the tail of the likelihood toward small masses $\left(M_{200} \sim 10^{11} M_{\odot}\right)$, which is due to the fact that subhalos with circular velocities as high as $v_{\text {circ }}=85 \mathrm{~km} \mathrm{~s}^{-1}$ are highly unlikely in such small host halos. As noted above, the lower velocity SMC does not influence the constraint due to the fact that the bulk of the constraint is due to the LMC that has the largest velocity. The addition of the SMC either as an independent tracer or as a second object in a pair to get the average center-of-mass values does not influence the constraint appreciably.

The summary of all the mass constraints is presented in Table 1. In the left half of the table we show the constraints obtained using host halos with any number of satellites with $V_{\text {circ }}>50 \mathrm{~km} \mathrm{~s}^{-1}$, while in the right half we show results obtained with the requirement that host halos host exactly two satellites with $V_{\text {circ }}>50 \mathrm{~km} \mathrm{~s}^{-1}$, as used in the study of B11. The first column lists the constraints for the $\mathrm{S}$ sample, for the case where we multiply the different likelihood distributions for each constraint instead of computing the mass likelihood directly (S-multi and LGP-multi), the case when only properties of the LMC are used in the constraint, and the case where we assume that the SMC and LMC are a bound pair and correspond to a single subhalo with properties given by the mass-weighted average of the SMC and LMC properties for the S and LGP samples (S-bound and LGP-bound). Columns 2-4 and 5-7 show the mass constraints and the errors corresponding to the $68 \%$ and $90 \%$ confidence intervals. $M_{200}$ masses can be converted to $M_{\text {vir }}$ using the conversion factor of $M_{\mathrm{vir}} / M_{200}=1.21$, computed directly by the Bolshoi simulation for the halos of this mass range.

\subsection{Close Satellite Pairs}

Satellite pairs are quite rare: we find that only $\approx 2 \%$ of the MW-sized halos have a pair of subhalos with $v_{\text {circ }}>50 \mathrm{~km} \mathrm{~s}^{-1}$, separation $<40 \mathrm{kpc}$, and relative velocity $<160 \mathrm{~km} \mathrm{~s}^{-1}$. This fraction drops to $\sim 1 / 30,000$ for pairs more closely resembling the SMC-LMC pair.

In this section, we explore the incidence of the population of close subhalo pairs and test whether their properties are distinct from the properties of the overall subhalo population. It is important to clarify these issues because velocities and other properties of the SMC and LMC may be influenced by their mutual interaction (e.g., D’Onghia \& Lake 2008; Nichols et al. 2011; Boylan-Kolchin et al. 2011). To select a sample of close pairs, we consider subhalos with $v_{\text {circ }}>50 \mathrm{~km} \mathrm{~s}^{-1}$ within the MW-sized halos that have distance to the host center, mutual separation, and absolute velocity similar to those of the MCs: $44 \mathrm{kpc}<r_{0}<66 \mathrm{kpc}, \Delta r<60 \mathrm{kpc}, 150 \mathrm{~km} \mathrm{~s}^{-1}<s<$ $400 \mathrm{~km} \mathrm{~s}^{-1}$, and $\Delta s<300 \mathrm{~km} \mathrm{~s}^{-1}$. We also estimate if the pairs are bound by computing the total energy assuming a two-body system of two point masses with masses of the SMC and LMC as derived from the $v_{\text {circ }}-M$ relation in the simulation. Such an approximation should be viewed as a rough approximation for the fraction of bound systems in a given sample, but can be used as a reference.

In Figure 7 we show the separation and relative velocities of the identified pairs (left panel) and velocity in the frame of the host halo of both pair members (right panel), with the $x$-axis showing velocity of the pair member with the larger circular velocity. The red points indicate pairs that are bound according to our energy estimate. The figure shows that only a small fraction of pairs has properties similar to those of the SMC-LMC pair $\Delta r_{\mathrm{MC}}=23.4 \pm 10 \mathrm{kpc}$, and $\Delta s_{\mathrm{MC}}=128 \pm 32 \mathrm{~km} \mathrm{~s}^{-1}$ (shown by the blue boxes in the two panels). The adopted range of separation of $23.4 \pm 10 \mathrm{kpc}$ is considerably larger than the actual observational error, which is of the same order as the error in the galactocentric distance: $\approx 2 \mathrm{kpc}$ (Kallivayalil et al. 2006a), but it allows us to select MC-like pairs without imposing a prohibitively restrictive constraint on the subhalo pair configuration. Only $\approx 60$ satellite pairs out of 1140 outside the green region have separations and relative velocities similar to those of the SMC and LMC, 90\% of which are bound according to our criterion.

The left panel of Figure 7 shows a rather large number of pairs clustered at separation $\lesssim 15 \mathrm{kpc}$ and $\Delta s \lesssim 70 \mathrm{~km} \mathrm{~s}^{-1}$. Most of these pairs appear to be artifacts due to failures of the halo finder for subhalo candidates close to the resolution limit of the simulation. We have visually inspected the dark matter density and velocity fields around a representative subset of these very close pairs and found that at separation $10-20 \mathrm{kpc}$, $\sim 20 \%$ subhalo pairs do not have corresponding distinct peaks in dark matter density field or coherently moving clumps of dark matter (i.e., subhalos are fake). Some of the pairs appear to be due to misidentification, in which a subhalo undergoing a tidal disruption is identified as two subhalos with circular velocities close to the resolution limit. On the other hand, we find that for pairs with separation $>20 \mathrm{kpc}$, almost all pairs have two clear distinct density peaks in the dark matter distribution and corresponding coherent velocity streams in the velocity field.

If we add the constraints that the pair of subhalos in host halos must have separation $\Delta r_{\mathrm{MC}}=23.4 \pm 10 \mathrm{kpc}$, and 

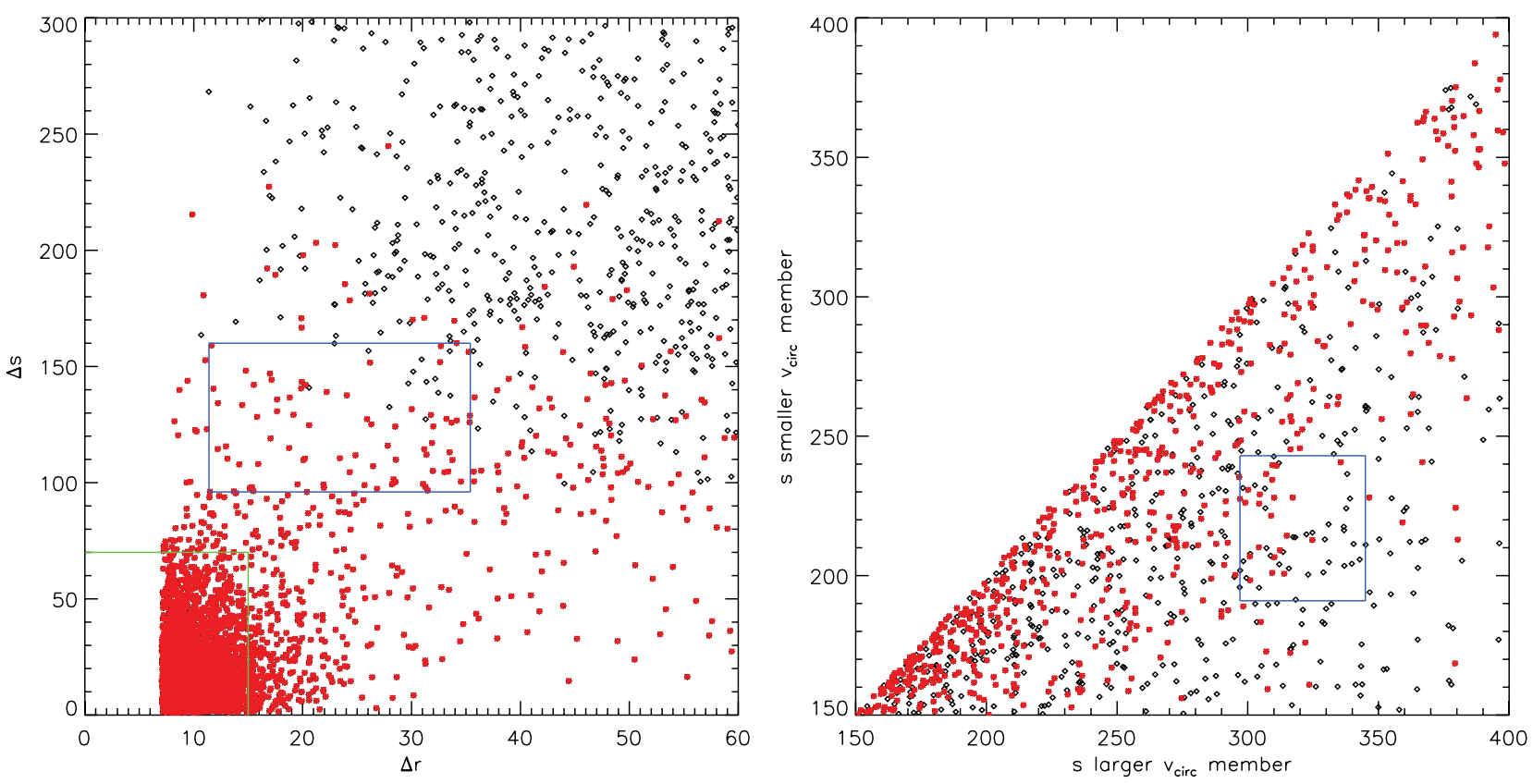

Figure 7. Properties of close subhalo pairs in MW mass halos. Left panel: pair separation and relative velocity; right panel: total speed relative to the host center for both pair members (horizontal axis for the member with larger circular velocity). The red points indicate subhalo pairs which are bound if we assume a two-body system. The blue rectangles show the MCs' constraints $\left(\Delta r<60 \mathrm{kpc}, \Delta s<300 \mathrm{~km} \mathrm{~s}^{-1}, 150 \mathrm{kms}^{-1}<\mathrm{s}<400 \mathrm{~km} \mathrm{~s}^{-1}, 44 \mathrm{kpc}<r_{0}<66 \mathrm{kpc}\right.$, and $\left.v_{\text {circ }}>50 \mathrm{~km} \mathrm{~s}{ }^{-1}\right)$. The green box in the left panel indicates very close pairs $\Delta r<15 \mathrm{kpc}, \Delta s<70 \mathrm{~km} \mathrm{~s}^{-1}$ which contain a large fraction of fake pairs (see the text for details).

(A color version of this figure is available in the online journal.)

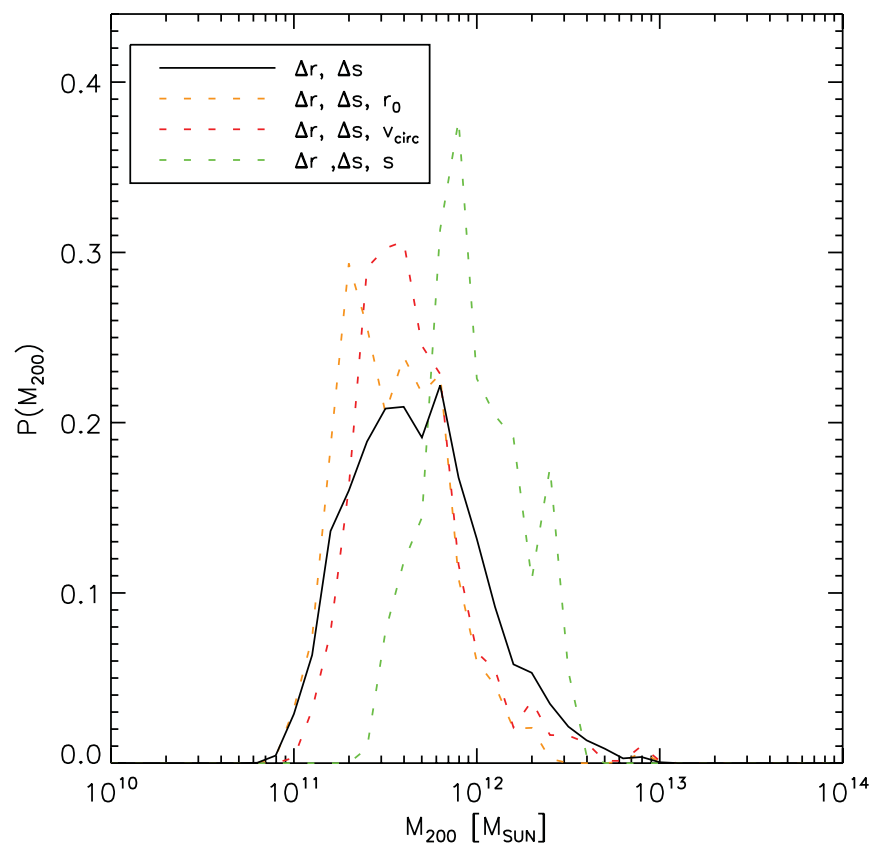

Figure 8. Likelihood distribution of the MW halo mass using additional constraints that subhalo pairs have separation and velocity difference similar to those of the SMC-LMC pair: $\Delta r_{\mathrm{MC}}=23.4 \pm 10 \mathrm{kpc}$ and $\Delta s_{\mathrm{MC}}=128 \pm$ $32 \mathrm{~km} \mathrm{~s}^{-1}$ (black solid line). Likelihood distributions for subhalo pairs matching only the radial distance (orange line), circular velocity (red line), or relative velocity with respect to the host halo (green line) are shown by the dotted lines. Less than $21 \%$ of close pairs are in halos of $M_{200}>10^{12} M_{\odot}$.

(A color version of this figure is available in the online journal.)

$\Delta s_{\mathrm{MC}}=128 \pm 32 \mathrm{~km} \mathrm{~s}^{-1}$, the resulting likelihood for the MW host halo mass is shown in Figure 8. Due to the small number of close subhalo pairs satisfying the constraints, the mass likelihood distribution can be computed using only one of the MCs' constraints at a time together with the separation and velocity difference constraints. We find that $<21 \%$ of close pairs are in halos of $M_{200}>10^{12} M_{\odot} \cdot{ }^{6}$ The addition of the constraint on the pair separation and relative velocity thus pushes the MW halo mass constraint to considerably lower masses, as compared to the constraint with only the properties used by B11. This illustrates that the actual constraint depends quite sensitively on which properties of the SMC-LMC system are chosen for the analysis, as is also clear from the large differences between mass likelihood distributions for the individual properties shown in Figure 5.

\section{DISCUSSION AND CONCLUSIONS}

In this study we have explored whether the environment of the MW affects the properties of its two most massive satellites, the SMC and LMC. These satellites are rather rare around galaxies with the MW luminosity and have rather high velocities. As argued by Busha et al. (2011a), the properties of the SMC and LMC can be used for a useful independent estimate of the MW virial mass. However, it would be straightforward to interpret such an estimate only if the MCs are not very unusual and their properties are not biased by the environment.

To test for the effects of the environment, we compare velocity distributions for a sample of MC-sized subhalos around MW hosts selected simply by mass and a sample of such halos selected with additional restrictions on the distance to the nearest cluster and local galaxy density, designed to mimic the environment of the LG. We find that the velocity distribution of satellites in the latter sample is shifted to somewhat larger velocities; in particular, the fraction of satellites with the LMC speed in the LGP sample is $23 \% \pm 2 \%$ larger than in the S sample. Thus, the LG environment enhances the probability of high satellite velocities, although the effect is mild.

\footnotetext{
6 This fraction is for the host halos restricted to have exactly two satellites with $V_{\text {circ }}>50 \mathrm{~km} \mathrm{~s}^{-1}$. If we allow any number of satellites, this fraction increases to $26 \%$.
} 
We compute the likelihood distribution for the MW halo mass using a method similar to that of Busha et al. (2011a), and explore how this distribution depends on different properties of the SMC and LMC used as constraints. We find no significant effect of the environment on the mass estimate. We also find that the treatment of different MC properties as mutually independent does not bias the mass constraint.

Using properties of the SMC and LMC from the recent study by Kallivayalil et al. (2013), we derive constraint on the MW halo mass of $\log \left(M_{200} / M_{\odot}\right)=12.06_{-0.19}^{+0.31}(90 \%$ confidence interval) for the $\mathrm{S}$ sample of halos selected without any environment restrictions. The mass constraint we derive is similar to that of B11, even though the updated values of MC properties, such as velocities and their errors, are quite different from the values used by B11. The mass constraint is broadly consistent with other recent estimates of the MW halo mass.

The method of B11 does therefore appear to be a robust way to measure the MW halo mass. A subtle issue, however, is that if some of the properties of the LMC or SMC used for the constraint are rare for the systems of the MW mass, the interpretation of the mass likelihood is not straightforward. The MW in this case may be located in the tail of the distribution and interpretation of the peak of the likelihood as most likely mass of the MW halo is not correct. It remains to be seen how rare particular properties of the LMC and SMC are. We do know, for example, that the probability of having two satellites of the SMC and LMC luminosity is by itself quite rare in the hosts of the MW luminosity (e.g., Liu et al. 2011; Robotham et al. 2012).

LMC and SMC are not just two unrelated luminous satellites: they are a close pair. We show in this study that close satellite pairs are quite rare: pairs with masses and separations similar to those of the LMC and SMC are found only in 1 out of $\approx 30,000 \mathrm{MW}$-sized halos. Observations also indicate that such close pairs are very rare (James \& Ivory 2011). We find that satellites in most close pairs with properties similar to the MCs are likely to be bound to each other. Interestingly, the halo mass likelihood distribution for host halos constrained to have MC-like close pairs of subhalos $\left(\Delta r_{\mathrm{MC}}=23.4 \pm 10 \mathrm{kpc}\right.$ and $\left.\Delta s_{\mathrm{MC}}=128 \pm 32 \mathrm{~km} \mathrm{~s}^{-1}\right)$ is quite different from the global likelihood from which the MW halo mass constraint discussed above was derived. In particular, less than $21 \%$ of host halos with $M_{200}>10^{12} M_{\odot}$ host MC-like close pairs. Taking into account the close separation of the MCs in the B11 method results in the shift of the MW halo mass estimate to smaller masses with the peak shifting approximately by a factor of two (see Figure 8). The reason for this shift is the fact that in smaller halos it is more likely to get a pair with small separation and relatively small velocity difference by chance (not necessarily bound), mainly because velocity dispersion is smaller in smaller mass halos.

This example clearly shows that a great care should be taken in choosing which of the satellite properties are used for the MW mass constraint.

This work was supported by the NSF via grant OCI-0904482. A.K. was in addition supported in part by the NSF grant AST-0807444 and by the Kavli Institute for Cosmological Physics at the University of Chicago through the NSF grants PHY-0551142 and PHY- 1125897 and an endowment from the Kavli Foundation. We have made extensive use of the NASA Astrophysics Data System and the arXiv . org preprint server.

\section{REFERENCES}

Besla, G., Kallivayalil, N., Hernquist, L., et al. 2007, ApJ, 668, 949 Besla, G., Kallivayalil, N., Hernquist, L., et al. 2010, ApJL, 721, L97 Besla, G., Kallivayalil, N., Hernquist, L., et al. 2012, MNRAS, 421, 2109 Boylan-Kolchin, M., Besla, G., \& Hernquist, L. 2011, MNRAS, 414, 1560 Boylan-Kolchin, M., Bullock, J. S., Sohn, S. T., Besla, G., \& van der Marel, R. P. 2013, ApJ, 768, 140

Bryan, G. L., \& Norman, M. L. 1998, ApJ, 495, 80

Bullock, J. S., Kravtsov, A. V., \& Weinberg, D. H. 2000, ApJ, 539, 517

Busha, M. T., Marshall, P. J., Wechsler, R. H., Klypin, A., \& Primack, J. 2011a, ApJ, 743, 40

Busha, M. T., Wechsler, R. H., Behroozi, P. S., et al. 2011b, ApJ, 743, 117

Casetti-Dinescu, D. I., Vieira, K., Girard, T. M., \& van Altena, W. F. 2012, ApJ, 753,123

Cuesta, A. J., Prada, F., Klypin, A., \& Moles, M. 2008, MNRAS, 389, 385

Deason, A. J., Belokurov, V., Evans, N. W., \& An, J. 2012, MNRAS, 424, L44

Diaz, J. D., \& Bekki, K. 2012, ApJ, 750, 36

Diemer, B., More, S., \& Kravtsov, A. 2013, ApJ, 766, 25

D’Onghia, E., \& Lake, G. 2008, ApJL, 686, L61

Evans, N. W., Wilkinson, M. I., Guhathakurta, P., Grebel, E. K., \& Vogt, S. S. 2000, ApJL, 540, L9

Font, A. S., Benson, A. J., Bower, R. G., et al. 2011, MNRAS, 417, 1260

Fouqué, P., Solanes, J. M., Sanchis, T., \& Balkowski, C. 2001, A\&A, 375,770

Gnedin, O. Y., Brown, W. R., Geller, M. J., \& Kenyon, S. J. 2010, ApJL, 720, L108

González, R. E., \& Padilla, N. D. 2010, MNRAS, 407, 1449

James, P. A., \& Ivory, C. F. 2011, MNRAS, 411, 495

Kafle, P. R., Sharma, S., Lewis, G. F., \& Bland-Hawthorn, J. 2012, ApJ, 761, 98

Kalberla, P. M. W., Dedes, L., Kerp, J., \& Haud, U. 2007, A\&A, 469, 511

Kallivayalil, N., van der Marel, R. P., \& Alcock, C. 2006a, ApJ, 652, 1213

Kallivayalil, N., van der Marel, R. P., Alcock, C., et al. 2006b, ApJ, 638, 772

Kallivayalil, N., van der Marel, R. P., Besla, G., Anderson, J., \& Alcock, C. 2013, ApJ, 764, 161

Karachenstev, I. D. 2012, AstBu, 67, 123

Karachentsev, I. D. 2005, AJ, 129, 178

Kauffmann, G., White, S. D. M., \& Guiderdoni, B. 1993, MNRAS, 264, 201

Klypin, A., Gottlöber, S., Kravtsov, A. V., \& Khokhlov, A. M. 1999a, ApJ, 516,530

Klypin, A., Hoffman, Y., Kravtsov, A. V., \& Gottlöber, S. 2003, ApJ, 596, 19 Klypin, A., \& Holtzman, J. 1997, arXiv:astro-ph/9712217

Klypin, A., Kravtsov, A. V., Valenzuela, O., \& Prada, F. 1999b, ApJ, 522, 82

Klypin, A. A., Trujillo-Gomez, S., \& Primack, J. 2011, ApJ, 740, 102

Komatsu, E., Smith, K. M., Dunkley, J., et al. 2011, ApJS, 192, 18

Koposov, S. E., Yoo, J., Rix, H.-W., et al. 2009, ApJ, 696, 2179

Kravtsov, A. 2010, AdAst, 2010, 8

Kravtsov, A. V., Gnedin, O. Y., \& Klypin, A. A. 2004, ApJ, 609, 482

Li, Y.-S., \& White, S. D. M. 2008, MNRAS, 384, 1459

Liu, L., Gerke, B. F., Wechsler, R. H., Behroozi, P. S., \& Busha, M. T. 2011, ApJ, 733,62

McConnachie, A. W., Irwin, M. J., Ferguson, A. M. N., et al. 2005, MNRAS, 356, 979

Mei, S., Blakeslee, J. P., Côté, P., et al. 2007, ApJ, 655, 144

Moore, B., Ghigna, S., Governato, F., et al. 1999, ApJL, 524, L19

Muñoz-Cuartas, J. C., Macciò, A. V., Gottlöber, S., \& Dutton, A. A. 2011, MNRAS, 411, 584

Nichols, M., Colless, J., Colless, M., \& Bland-Hawthorn, J. 2011, ApJ, 742,110

Nulsen, P. E. J., \& Bohringer, H. 1995, MNRAS, 274, 1093

Olsen, K. A. G., Zaritsky, D., Blum, R. D., Boyer, M. L., \& Gordon, K. D. 2011, ApJ, 737, 29

Ribas, I., Jordi, C., Vilardell, F., et al. 2005, ApJL, 635, L37

Robotham, A. S. G., Baldry, I. K., Bland-Hawthorn, J., et al. 2012, MNRAS, 424,1448

Schaap, W. E., \& van de Weygaert, R. 2000, A\&A, 363, L29

Smith, M. C., Ruchti, G. R., Helmi, A., et al. 2007, MNRAS, 379, 755

Sofue, Y., Honma, M., \& Omodaka, T. 2009, PASJ, 61, 227

Tollerud, E. J., Boylan-Kolchin, M., Barton, E. J., Bullock, J. S., \& Trinh, C. Q. 2011, ApJ, 738, 102

Wang, J., Frenk, C. S., Navarro, J. F., Gao, L., \& Sawala, T. 2012, MNRAS, 424,2715

Watkins, L. L., Evans, N. W., \& An, J. H. 2010, MNRAS, 406, 264

Wilkinson, M. I., \& Evans, N. W. 1999, MNRAS, 310, 645

Xue, X. X., Rix, H. W., Zhao, G., et al. 2008, ApJ, 684, 1143 